\title{
Civilisations
}

Revue internationale d'anthropologie et de sciences

humaines

$41 \mid 1993$

Mélanges Pierre Salmon II

\section{Les perles au-delà du décoratif dans le golfe du Bénin à travers les âges}

\section{A. Félix Iroko}

\section{OpenEdition}

\section{Journals}

Édition électronique

URL : http://journals.openedition.org/civilisations/1700

DOI : 10.4000/civilisations. 1700

ISSN : 2032-0442

\section{Éditeur}

Institut de sociologie de l'Université Libre de Bruxelles

\section{Édition imprimée}

Date de publication : 1 septembre 1993

Pagination : 145-164

ISBN : 2-87263-094-5

ISSN : 0009-8140

Référence électronique

A. Félix Iroko, "Les perles au-delà du décoratif dans le golfe du Bénin à travers les âges », Civilisations [En ligne], 41 | 1993, mis en ligne le 28 juillet 2009, consulté le 02 mai 2019. URL : http:// journals.openedition.org/civilisations/1700 ; DOI : 10.4000/civilisations.1700

Ce document a été généré automatiquement le 2 mai 2019.

(c) Tous droits réservés 


\section{Les perles au-delà du décoratif dans le golfe du Bénin à travers les âges ${ }^{1}$}

\section{A. Félix Iroko}

1 La portion des côtes ouest-africaines qu'occupe le Golfe du Bénin va de la Volta au Ghana jusqu'au delta du Niger à l'Est, au Nigéria. Elle regroupe, placée à l'échelle de la géopolitique actuelle, une partie de la côte ghanéenne, tout le littoral togolais, béninois et une portion du Nigéria méridional. Ce Golfe rassemble un certain nombre de groupes socio-culturels aux cultures matérielles diverses. Parmi celles-ci, les perles, souvent citées dans les documents anciens et les sources orales pour leur fonction de parures, n'ont jamais fait l'objet d'une étude d'ensemble mettant en relief leurs différents usages et leur place dans les représentations collectives et l'univers conceptuel des habitants du Golfe du Bénin. L'accent a souvent été mis sur l'aspect décoratif, c'est-à-dire sur la vocation esthétique de ces perles au détriment de ce qu'elles représentent de beaucoup plus profond, de beaucoup plus intime pour les Africains dans leurs attitudes collectives, leurs structures mentales, leur réflexion sur la vie, sur les hommes. Nous voudrions montrer ici qu'en dehors du décoratif, les perles ont, dans les civilisations africaines, d'autres usages plus diversifiés et parfois si discrets qu'ils peuvent se dérober aux investigations du chercheur peu attentif, peu averti.

\section{Les perles dans le domaine des croyances populaires et du religieux}

2 Les perles, qu'elles soient de verre, de pierre ou recueillies sur les côtes Ouest-africaines ${ }^{2}$, n'ont en elles-mêmes rien de mystérieux, de divin ou de sacré. Or, leur nature, la difficulté de déterminer la provenance de certaines d'entre elles, les vertus magiques que l'imagination leur attribue, ont conduit les Noirs du Golfe du Bénin à trouver une origine divine à plus d'une variété d'entre elles ${ }^{3}$, et à leur faire un traitement de choix dans leurs croyances populaires. Jean Hess a recueilli au XIX ${ }^{\mathrm{e}}$ siècle un mythe yoruba faisant état des conditions de création des perles par Olodumaré le Tout-Puissant : 
"... Au commencement, la terre n'existait pas... Et aucun être n'animait le ciel, n'animait l'eau. Or, le Tout- Puissant Olodumaré, le Maître et le Père de toutes choses, qui habite là-haut, derrière les montagnes, bien loin, très haut, plus haut que personne ici-bas ne peut se le figurer... résolut un jour de créer. Il créa d'abord les sept princes couronnés. Pour la nourriture et l'entretien de ces princes, il créa ensuite sept calebasses très grosses et pleines d'akassas, et sept sacs dans lesquels il $\mathrm{y}$ avait des cauris, des perles et des étoffes ${ }^{4}$.

3 Les Ewé du Ghana et du Togo méridionaux ainsi que les groupements socio-culturels apparentés considèrent Aydohouèdo, symbolisé par l'Arc-en-ciel le Grand Serpent, comme la divinité et des perles et des cauris, bref comme l'une des grandes divinités de la richesse $^{5}$. Aussi a-t-elle toujours été en honneur chez eux, notamment durant la période précoloniale au cours de laquelle A.B. Ellis écrivait à ce sujet les lignes suivantes :

"Popo beads, the aggry-beads of the gold Coast, curious mosaico vvhose origin in undetermined, and which are much valued by the Negroes, are believed to be made by Anyi-ewo [Ayido-Houèdo]. This excrement is believed to have the power of transmuting grains of maize into cowries. Whence the notion commonly held by Europeans in West Africa, that Anyi-ewo confers wealth on man"6.

Les Hula ou Popo du Mono, en République du Bénin, prétendent également que les perles jaunes enjolivées de stries de couleur sont l'oeuvre du serpent Dan-Aïdo-Ouèdo ${ }^{7}$, l'Esprit de l'Arc-en-ciel, d'où le nom de perles addo qu'ils leur donnent ${ }^{8}$. "Ayido-whe-Do qui a fait des perles popo", écrivait déjà, dans la deuxième moitié du XIX ${ }^{\mathrm{e}}$ siècle, l'Anglais Sir Richard Francis Burton'. L'on comprend que désirant enrichir un individu, Dan le serpent, en liaison avec l'Arc-en-ciel (en pays fon) le conduise par une force aveugle à l'endroit où la queue de ce dernier est censée toucher la terre ${ }^{10}$. Il s'y produit alors une profonde excavation renfermant de l'or et des perles ${ }^{11}$.

Le Révérend Père Jacques Bertho a recueilli des traditions relatives aux perles qui dans l'esprit des populations locales, ne seraient que des excréments de divinité, du "serpent fétiche" comme il l'écrit ${ }^{12}$. Il s'agit, encore une fois, de Dan-Ahido-Houèdo, (Bertho) l'arcen-ciel dont les pythons seraient les messagers. Il vivrait sous terre, mais parfois en sortirait au milieu de la forêt, "s'élèverait dans les airs pour aller plonger dans la mer afin d'y étancher sa soif. A l'endroit où il est sorti de terre, il laisse des excréments qui sont des perles précieuses"13. Les divinités Aziza, détentrices des vertus médicinales des plantes, se chargeraient, une fois Dan-Ahido-Houèdo sorti de terre, de recueillir les perles - ses excréments - et de les enfouir dans le sol pour les sauvegarder ${ }^{14}$.

6 Ce regard mythique que les populations du Golfe du Bénin projettent sur les perles qu'elles considèrent comme sacrées, voire divines, permet à lui seul de comprendre le bien-fondé de maints usages de ces précieux objets en matière de pratiques religieuses et magiques : aussi prêtres et adeptes de divinités portent-ils des colliers, des bracelets de perles ainsi que des filières de perles en bandoulières; la nature de ces perles, leur variété, leur coloris, leur style d'agencement, la manière dont elles sont portées variaient beaucoup, et de façon remarquable, d'une secte religieuse à une autre ${ }^{15}$. Sacrées et dotées de vertus magiques de toutes sortes, ces perles portées par les adeptes de différentes confréries religieuses étaient aussi un signe de distinction et de différenciation de ces communautés religieuses. Aussi, les perles sont-elles toujours choisies en harmonie avec le goût de la divinité dont relève la secte : ce sont des perles spéciales portées sous forme de colliers qui constituent le pouvoir et l'insigne du pouvoir de Gangnihesu ${ }^{16}$ à HuawéZunzonsa (région d'Abomey) où il exerce ses fonctions de grand prêtre sacrificateur $\mathrm{d}^{\prime}$ 
Aïzan Daho ${ }^{17}$, divinité autour de laquelle les princes se réunissent périodiquement pour des cérémonies religieuses.

7 En pays yoruba, une enfilade de perles dites ilèkè otutu okpun au poignet gauche du babalawo - devin d'Ifa - constitue l'insigne de ses pouvoirs occultes et le distingue quelque peu du commun des mortels qui n'est pas autorisé à en porter.

8 En pays aja dans le Mono béninois, il y a également des variétés de perles qui interviennent dans la pratique oraculaire $f a$; ce sont des perles jaunes à longue perforation dites afasi-kuè ou monnaies de $\mathfrak{f a}^{18}$. Il est d'autres variétés de perles qui, au lieu d'être utilisées telles quelles dans le fa comme les afasi-kuè, sont écrasées et réduites en poudre étalées par la suite sur le plateau divinatoire pour y recevoir les différents signes divinatoires auxquels elles confèrent davantage de puissance pour conjurer le mauvais sort ou guérir une maladie.

Spécialement apprêtées en matière d'occultisme, certaines perles servent à la prophylaxie ou au traitement des maladies, ainsi qu'à la protection contre les morsures de serpent, les sorciers, les mauvais esprits ${ }^{19}$ et, d'une façon générale, contre le mauvais oeil. Il en est ainsi dans le Golfe du Bénin comme ailleurs en Afrique noire. Nous retrouvons ce même usage en Afrique du Nord où D. Champault et $\mathrm{M}$. de Langle ont vu des enfants porter des perles pour se prémunir contre "le mauvais oeil" et l'action maléfique d'esprits malins ${ }^{20}$.

10 Quiconque porte sur lui la perle nana cylindrique, bleu-azur plus ou moins translucide ${ }^{21}$, magiquement préparée, ne périra jamais dans un incendie ou dans un feu quelconque, car cette variété de perles, très prisée, a la caractéristique d'être résistante au feu qui ne parvient jamais à la détruire ou à l'altérer. Très classique dans les aires culturelles du Golfe du Bénin, une telle analogie n'en est pas moins frappante.

11 Réserve de valeur sur le plan monétaire puisqu'elles se prêtent à merveille à la thésaurisation, les perles, denrée non périssable, participent aussi à la thésaurisation funéraire à l'instar des cauris ; ainsi, de petites urnes funéraires en céramique contenant les perles les plus précieuses sont placées dans les tombes dans l'intention de constituer pour les morts de véritables réserves de numéraire et de parures pour l'au-delà. Edouard Dunglas, admnistrateur des Colonies, a noté, lors de son séjour au Dahomey ${ }^{22}$, une telle pratique, courante chez les Fon d'Abomey. Il arrive dans certaines régions des aires culturelles ajatado et yoruba que des cauris soient associés à des perles dans une urne funéraire ou mis dans un autre vase à côté de celui des perles. De façon générale, celles-ci, dans le cas d'une femme défunte, constituent, en partie ou en totalité, les richesses qu'elles possédaient de son vivant ${ }^{23}$; il s'agit donc, en général, de perles de son goût, ce dernier ne variant pas, pense-t-on, dans l'autre monde ${ }^{24}$. Bien "des gisements" de perles aujourd'hui mis au jour lors des travaux agricoles, de terrassements ou de fouilles archéologiques ne sont rien d'autres que le trésor des morts.

Les perles provenant de la thésaurisation funéraire sont particulièrement recherchées dans des pratiques occultes de toutes sortes, car leur contact avec les morts a dû, pense-ton, accroître le pouvoir qui était naturellement le leur; aussi coûtent-elles toujours beaucoup plus cher que les perles qui n'ont circulé que parmi les vivants ${ }^{25}$. Elles ont en général des fonctions ambivalentes: destinées le plus souvent à des pratiques occultes pour le bien-être des hommes, elles servent également à nuire dans des circonstances particulières, où l'on dépose discrètement ces objets maléfiques dans les effets d'un 
individu en priant le mort dépossédé de venir chercher son bien volé en se faisant accompagner - sans retour naturellement - par celui qui est en sa possession.

Par ailleurs, les perles ont une place non négligeable dans l'anthropologie des naissances géméllaires: les statuettes de bois qui, dans les aires culturelles ajatado et yoruba remplacent les jumeaux défunts, portent parfois des colliers ou des bracelets de perles ${ }^{26}$. Chez les Guin ou Mina d'Aného, des mères de jumeaux ou de plusieurs enfants (au moins trois) de même sexe nés successivement sans qu'un enfant d'un autre sexe s'intercale entre eux, portent le abahun, bracelet garni d'une perle bleue ${ }^{26}$. Bien des variétés de perles, prescrites par l'oracle $\boldsymbol{f} \boldsymbol{a}$ ou $\boldsymbol{i} \boldsymbol{f a}^{27}$, sont spécialement apprêtées et portées par les jumeaux pour leur protection ou pour leur enrichissement, surtout lorsqu'ils sont nés sous le signe de la richesse.

\section{Les perles, monnaie et symbole de richesse}

Lorsqu'il est question de perles, l'idée qui vient d'emblée à l'esprit de quiconque est celle de parures, et presque jamais, sinon secondairement, celle de monnaie. Pourtant, en bien des endroits des côtes ouest-africaines, les perles ont eu une véritable fonction monétaire : intermédiaire dans les échanges, elles étaient également un étalon de valeur de par la référence commune qu'elles étaient, et une réserve de valeur autorisant la thésaurisation, aussi bien au profit des vivants que des morts ${ }^{28}$.

Cette fonction d'équivalent général n'a jamais été, bien entendu, un phénomène généralisé, d'une grande ampleur chronologique et d'une étendue géographique significative susceptibles d'être comparées à celles des cauris notamment; mais des îlots de "zone perle" souvent au milieu d'autres espaces monétaires plus vastes comme la "zone or" et la "zone cauris"29 ont existé dans le Golfe de Guinée durant la période précoloniale. A la Côte des Esclaves alors fortement dominée en matière monétaire par une circulation des cauris qui, des siècles durant, avait été la plus forte au monde, des perles ont également, mais à une échelle très restreinte, circulé comme équivalent général.

16 Le père Loyer, missionnaire à Assinie en Côte-d'Ivoire au XVIII ${ }^{\mathrm{e}}$ siècle, écrivait que les perles d'Aigris y servaient de monnaie courante ${ }^{30}$. Deux siècles plus tard, l'administrateur français Maurice Delafosse, qui a séjourné des années durant en Côte-d'Ivoire, a rapporté que des perles, en masse, en filière, en collier, en ceinture, étaient utilisées comme médium dans les échanges commerciaux en pays agny ${ }^{31}$.

17 Les perles étaient si valorisées que Duarte Pacheco Pereira, gouverneur du Fort de la Mine au Ghana de 1520 à 1522, a constaté que les bleues à lignes rouges appelées cori y étaient vendues contre de l'or ${ }^{32}$. Au XVII siècle, le Bâlois Samuel Braün écrivait que ces pierres bleuâtres transportées dans le Golfe de Guinée se vendaient fort cher et étaient évaluées littéralement à leur poids d'or ${ }^{33}$. "Dans l'ancien système monétaire local, elles étaient aux cauris ce que les pièces d'or étaient aux gros sous" écrivait Edouard Dunglas ${ }^{34}$.

Les perles étaient donc considérées comme des produits extrêmement précieux : articles privilégiés de commerce, elles se vendaient et s'achetaient cher, tout en servant localement comme équivalent général à l'acquisition d'autres articles, avec, bien entendu, le statut privilégié d'une denrée précieuse de référence. En outre, apprêtées dans des conditions occultes particulières, elles sont souvent discrètement utilisées par des marchands qui les placent dans leurs porte-monnaies pour protéger leur avoir du regard 
rapace de ceux à qui l'on prête le pouvoir de voler l'argent d'autrui rien qu'en le regardant attentivement ${ }^{35}$.

Une variété de perles que nous n'avons pu identifier aurait même naturellement le pouvoir d'annihiler l'effet de la "monnaie de la ruine" destinée à entraîner la faillite de marchands chez qui on a acheté un article avec une telle monnaie ${ }^{36}$.

Toutes ces raisons jointes aux mythes archétypaux d'origine des perles, permettent de comprendre pourquoi elles sont devenues dans l'esprit des habitants du Golfe du Bénin, le symbole même de la richesse et de sa conservation au même titre que les cauris qui y ont eu pourtant un rôle monétaire beaucoup plus important. Avoir des perles en abondance, c'est être riche ; aussi, un important clan de l'aire culturelle ajatado porte-t-il avec fierté le nom de Jèto ou cours d'eau des perles en souvenir de l'ancêtre qui, dit-on, possédait une rivière où il allait ramasser à volonté des perles ${ }^{37}$, sous le regard envieux des gens de son milieu.

La notion de richesse incarnée par les perles a également une autre dimension symbolique qui n'est pas toujours ni nécessairement fonction de leur quantité : appelé dada - Sa Majesté - le roi du Danhomè dans ses louanges est habituellement désigné, entre autres, par le nom jêhosu, c'est-à-dire le roi des perles, non pas nécessairement à cause de l'abondance de perles précieuses contenues dans ses coffres, mais parce qu'il était plus riche que quiconque dans son royaume: abondance de cauris, de tissus, de métaux précieux, de verroteries, de biens meubles, etc. De nombreux auteurs ou voyageurs ayant séjourné dans le royaume de Danhomè, ont porté des témoignages élogieux sur les richesses des souverains du Danhomè: l'Anglais Bullfynch Lamb, prisonnier du roi Agaja dans le premier quart du XVII ${ }^{e}$ siècle, envoya au Gouverneur Tinker le 27 novembre 1724, une lettre dans laquelle il donne une idée de l'impressionnante richesse de ce souverain conquérant :

"Je ne vois d'autre moyen de me racheter, sinon que la Compagnie lui envoye en présent une couronne et un sceptre dont elle [Sa Majesté] pourrait prendre la valeur sur ce qui reste dû au dernier roi d'Ardah. Je ne sais quel autre présent lui faire qu'il ne le dédaigne, car il est très fourni de quantités de vaisselles d'or en oeuvre, et de toutes sortes de richesses, aussi bien que de beaux habillements, chapeaux, bonnets, de toute espèce : il abonde en outre en marchandises dont il ne lui manque aucune : il donne des Boojes, Buyis (cauris) comme de la poussière, et il distribue des liqueurs comme de l'eau. Il est extrêmement vain et présomptueux, et je crois en effet qu'il est le Roi le plus riche et le plus grand guerrier dans cette partie du monde....

C'est en fait beaucoup plus l'abondance et la qualité de tous ses biens qui sont indirectement rappelées à travers le nom jêhosu ${ }^{39}$ donné au roi du Danhomè, qu'un éventuel et inégalable volume de perles qu'il posséderait.

Assimilées ailleurs et dans d'autres circonstances à la richesse par excellence quand elles sont abondantes, les perles, même absentes donnent leur nom à toutes richesses comme dans le cas des souverains du Danhomè. Les perles incarnent la richesse; la richesse incarne indirectement les perles en ce sens qu'elle est supposée être aussi précieuse et aussi difficile à acquérir qu'elles. La nuance, aussi ténue soit-elle, a son prix et elle était toujours présente dans l'esprit des habitants du Golfe du Bénin quand ils apprécient diversement les perles, aussi valorisées quand elles existent abondamment que dans le cas où, absentes, leur silhouette est supposée se profiler à l'arrière-plan de tout tableau de grandes richesses, même dans les réalités politiques comme à Abomey: il y a, en quelque sorte le pouvoir des perles et les perles du pouvoir. 


\section{Les perles, signes distinctifs et insignes du pouvoir} dans les civilisations yoruba ou nago, qu'elle concerne les ministres à la cour ou la royauté proprement dite incarnée par le souverain. Le plus important des régalia, de façon générale dans l'aire culturelle yoruba incluant les Nago, ce ne sont point seulement les sandales comme dans le Danhomè, mais bien plutôt le couvre-chef de perles garni par devant de multiples filières ou franges de perles multicolores ${ }^{40}$, en rangées si serrées qu'elles laissent à peine apparaître le visage du souverain qui le porte ${ }^{41}$; et ces franges sont agencées si étroitement à dessein qu'elles avaient effectivement pour fonction de dérober les traits du visage du souverain au regard du public ${ }^{42}$. Le roi se cache en quelque sorte derrière "ce masque", cette forêt de perles agencées avec un soin qui ne manque pas d'esthétique bien que cette dernière préoccupation soit tout à fait secondaire ici dans les fonctions auxquelles est destiné le couvre-chef. Ce dernier, qui tient ici lieu de couronne ${ }^{43}$ en quelque sorte, constitue l'élément de référence en matière de typologie des rois en pays yoruba: ceux d'entre eux qui portent ce couvre-chef de perles sont les plus importants, les rois par excellence. Ils prennent naturellement le pas sur les souverains qui ne sont pas autorisés à avoir de couvre-chef de perles ${ }^{44}$. Les rois-prêtres (ou les prêtres-rois ?) de Pobè (République du Bénin) qui se ceignent la tête d'un tissu blanc sont considérés comme inférieurs aux rois de Kétu, d'Ifè, d'Oyo, etc. qui, eux, portent des couronnes perlées garnies de filières de perles multicolores.

De façon générale, l'insigne de commandement des rois de Kétu (alakétu) est une queue à manche entièrement entourée de perles fines et multicolores très joliment agencées qui, dit-on confère à cet attribut du pouvoir toute sa puissance. L'on raconte à ce sujet que l' alakétu Odémufèkun se rendait un jour en hamac de Kétu à Zagnanado. C'était en 1930. En chemin, il vit une antilope sortir de la brousse. Il tendit son orukè (cette queue à manche recouverte de perles) dans sa direction en lui intimant l'ordre de s'arrêter sans plus 
bouger. L'antilope s'était aussitôt immobilisée sous l'influence magique conjuguée des incantations du roi et de l'orukè qui agissait comme une baguette magique.

Les queues de cheval qui servaient de chasse-mouches à des rois avaient leurs manches entourés de perles pour, dit-on, renforcer leur pouvoir ${ }^{45}$. De même, les bâtons des messagers royaux de Kétou étaient entièrement recouverts de perles.

Dans les cours royales yoruba ou nago, les ministres des rois portaient des colliers ou des bracelets de grosses perles, insignes de leur dignité. Ces perles dont la nature et le nombre variaient selon l'importance de chaque ministre et sa place à la cour, n'avaient, contrairement aux perles minuscules des couvres-chefs et des sceptres des souverains, aucun - sinon très peu - de pouvoir magique ${ }^{46}$. Elles n'étaient généralement que des signes distinctifs permettant de ne pas confondre les ministres qui les portent. Afolabi Ojo, dans son excellente étude sur la culture yoruba, résume en des termes saisissants les fonctions discriminatoires et distinctives attribuées aux perles qui garnissent les regalia et complètent l'accoutrement des souverains et de leurs ministres dans les royautés yoruba ; il écrit notamment à ce sujet :

"The bead crafts developed as a result of the effective demand made by chiefs and Obas for beaded objects which, more than any other, dominated their regalia. The wearing of beads distinguished chiefs and Obas in an unmistakable manner from the non-titled person, just as badges and other insignia indicate rank. Significant too was he part of the body on which the beads were worn. Crowned obas not only wore beaded crowns with fringes but also strings of the barrel-shaped and round beads around the neck, wrists and around and ankles. A village chief was not entitled to wear a beaded crown but migth wear beads around the neck, wrists and ankles. Many ordinary chiefs wore beads around the neck and wrists only, the number of strings and the shape reflecting their rank" ${ }^{147}$.

Ces perles qui sont soit de verre, soit de cernaline, soit de corail, soit de quartz, soit d'autres matières, ne sauraient être utilisées par des profanes pour les mêmes préoccupations politiques sous peine de sanctions de la part du pouvoir central. Nul, dans la société yoruba, n'a le droit de porter un couvre-chef de perles à l'instar du roi. Il était, également, interdit de porter aux poignets et au cou les mêmes perles que les ministres ou dignitaires de la cour. Ce sont là les principales fonctions ou attributs politiques des perles qui, malgré leur importance, sont loin d'être aussi étendues que leurs fonctions sociales.

\section{Fonctions sociales profanes des perles}

31 Les fonctions sociales à caractère profane des perles sont multiples et elles le sont autant que les autres fonctions; néanmoins, nous renonçons dans le cadre de cette approche rapide, à l'étude de leur rôle de décoration, de parure qui, sans manquer d'intérêt, n'a plus aujourd'hui l'avantage de la nouveauté, l'Afrique n'ayant pas par ailleurs le monopole de l'exclusivité en ce domaine. En revanche, nous insisterons sur la place accordée aux perles dans le domaine de l'amour. En effet l'un des canons de beauté chez les femmes de la plupart des groupes ethniques du Golfe du Bénin consistait à porter des filières de perles aux jarrets et aux reins. Malgré l'harmonie de l'agencement de ces perles, leur fonction n'est jamais prioritairement l'esthétique, mais l'érotisme : elles sont essentiellement destinées à exciter la sensualité, l'appétit sexuel des hommes qui savent les apprécier à leur juste valeur : les jarretières de perles donnent davantage de relief aux mollets que les hommes éprouvent du plaisir à caresser en même temps que les perles 
multicolores ${ }^{48}$. Quant aux perles portées aux reins, elles sont de taille extrêmement variable: des plus fines aux plus grosses en passant par celles de taille moyenne. Cependant, les plus appréciées étaient celles qui sont dites bébé ou abébé dans les aires culturelles yoruba et ajatado : ce sont des perles discoïdes minces et plates, à très courte perforation centrale de couleur noire, bleue ou rouge; elles étaient assez fragiles et ne résistaient pas à l'épreuve du feu comme les nana. Il en fallait un très grand nombre pour une rangée destinée à faire le tour de ceinture d'une femme. Enfilées, elles se présentent sous l'aspect d'une grosse ceinture. Selon leur goût, les femmes peuvent enfiler soit exclusivement des perles noires, soit des perles rouges, soit des bleues, soit panacher les trois. De même, elles peuvent porter soit uniquement des enfilades d'une seule couleur, soit des rangées de chacune des couleurs. Certaines cependant se contentent d'enfilades d'un seul coloris non pas pour une question de goût, mais à cause des interdits en liaison avec leur destinée ${ }^{49}$.

Discrètement portées sous leurs pagnes par les femmes ${ }^{50}$, ces grosses rangées de perles donnent de l'extérieur plus d'embonpoint à leur hanche, ce qui est très apprécié dans ces milieux où la stéatogypie est perçue comme un des éléments d'attrait d'une femme. En outre, dans des conditions d'intimité avec un homme, celle qui porte ces rangées de perles est censée être plus sensuelle que celle qui en est dépourvue. Jusque dans la première moitié $\mathrm{du} \mathrm{XX}$ siècle, il était rare qu'une femme ne porte pas de perles sous ses pagnes. Cela a inspiré les Fon dans l'une de leurs manières de dire qu'un homme s'est rendu coupable d'adultère avec une femme: é ha jè do alin ni: littéralement: il lui a compté les perles aux reins. Cette forme d'euphémisme découle du fait qu'il faudrait être dans la plus grande intimité avec une femme pour être en mesure, non pas seulement de voir les perles qu'elle a aux reins sous ses vêtements et les toucher, mais aussi de les compter. Cela ne signifie nullement qu'avoir des relations intimes coupables avec une femme passe par le dénombrement des innombrables perles des enfilades qu'elle porte mais c'est une manière plus élégante d'exprimer un adultère, en se fondant sur le port de perles par les femmes.

33 Par ailleurs, fines, moyennes ou grosses, les enfilades de perles portées sous leurs pagnes par les femmes jouent un autre rôle : elles servent a retenir par devant et par derrière les deux bouts des couches hygiéniques dont elles "se protègent" lors des périodes de menstrues.

Dans un autre ordre d'idées, l'on ne saurait faire une étude exhaustive de la musique traditionnelle du Golfe du Bénin sans y mettre en relief le rôle des perles : des enfilades de ces dernières harmonieusement agencées entourent amplement des gourdes, de manière à ce qu'elles produisent des sons en tapant sur les parois de cette dernière lorsqu'elle est rythmiquement secouée ${ }^{51}$.

Aucune étude n'a jusqu'ici porté sur la place des perles dans la littérature orale traditionnelle des sociétés du Golfe du Bénin : nous n'insisterons plus sur le nom du clan dénommé jêto (cours d'eau des perles) dont nous avons déjà parlé plus haut dans la deuxième partie de cette approche ; le clan continue toujours de porter fièrement ce nom, bien qu'il n'ait plus aujourd'hui le moindre lien avec un quelconque point d'eau à perles. Par ailleurs, en liaison avec les perles, l'anthroponyme le plus connu dans l'histoire du royaume du Danhomè est $\mathrm{Na}$ Zognidi épouse du roi Guézo et mère de son fils Glèlè. Ce nom fut donné à cette reine en fonction des innombrables épreuves qu'elle a su traverser saine et sauve à la manière de la perle nana toujours résistante au feu ${ }^{52}$. 
Les Noirs du Golfe du Bénin ont aussi trouvé des sources d'inspiration dans l'usage des perles pour élaborer leurs proverbes et dictons ; c'est ainsi que les Gun de Porto- Novo pour signifier qu'il ne suffit pas de vouloir quelque chose pour être en mesure de l'avoir disent souvent: "aco jro gidi ; yonu ma diké"53 : le singe voudrait bien se parer de beaux atours, porter autour des reins des rangées de perles mais il lui manque l'attribut principal : les fesses"54. Quant aux Fon d'Abomey, ils recommandent la patience en toutes choses en utilisant le proverbe suivant: "Dada Sègbo so lankan gban nyi do ji, bo hué su lo e no wen dokpo"55, "Dieu accrocha au firmament 30 perles précieuses lankan et en décroche une par an"56.

7 Les exemples de la place des perles dans la littérature orale traditionnelle peuvent être multipliés presque indéfiniment à partir de la constitution minutieuse d'un riche corpus d'anthroponymes, de maximes, de dictons populaires, d'adages: de véritables trésors d'imagination et de composition chez les peuples du Golfe du Bénin.

\section{Conclusion}

8 Les marchands européens qui, durant la période précoloniale, vendaient aux Noirs du Golfe du Bénin des perles importées de chez eux ou d'autres points d'Afrique, ne s'imaginaient nullement qu'elles pouvaient servir à d'autres fins qu'esthétiques. Or, les Africains les ont intégrées à leurs cultures en allant au-delà du superficiel, du visible immédiat que constitue le décoratif dont l'étude n'a plus aujourd'hui le charme de la nouveauté, de l'inédit ou de la contribution.

Saisies de l'intérieur, les différentes fonctions non décoratives des perles sont riches de perspectives et d'axes de réflexion pour le chercheur qui tente de cerner de très près ce que représentent de plus profond et de plus intime pour les Africains: un filon pour l'historien des mentalités, l'ethnologue et l'anthropologue des cultures en liaison avec les structures mentales.

- Cette approche introductive rapide qui n'a d'autres ambitions que celle de susciter un débat autour d'une donnée apparemment anodine, fait entrevoir tout ce qu'il y a encore à faire sur ces petits objets en matière de symbolique et d'attitudes collectives des populations. Les études ultérieures gagneront cependant à être moins globalisantes et plus fines : il s'agira pour elles, entre autres, de répertorier toutes les variétés anciennes de perles ainsi que leurs noms dans les langues des milieux concernés par leurs usages ; de recueillir tous les mythes et autres représentations de mentalités dont elles sont chacune l'objet; les différents usages auxquels elles étaient et sont encore destinées; d'insister sur les éléments ou facteurs de permanence ou de rupture dans leurs usages ou leurs perceptions, les diversités d'un espace à l'autre de leurs utilisations. Il n'est pas exagéré de dire que les perles sont un véritable fait de civilisation en Afrique, d'où tout l'intérêt de leur étude qui attend d'être réalisée sur une grande échelle à partir de très modestes monographies. 


\section{NOTES}

1. La bibliographie sur les perles dans le Golfe du Bénin est relativement abondante. Nous nous contenterons de citer pour mémoire quelques titres pouvant permettre au lecteur de se faire une idée des travaux sur la question.

- FORBES (F.E.) : Dahomey and the Dahomans. Being the Journals of two missions to the King of Dahomey and residence at his capital in the years (1849-1850). London, Routledge and Kegan Paul, 1864 (Réédition de 1966, 372 p.).

- BURTON (Sir R.) : A Mission to Gelele king of Dahome. London, Routledge and Kegan Paul, 1864 (Réédition de 1966, 372 p.).

- ELLIS (A.B.) : The Ewe-Speaking peoples of the Slave Coast of West Africa: their religion, manners, customs, laws, languages, etc. London, Chapman and Hall, 1890, 331 p., 1 carte.

- HESS (J.) L'âme nègre.Paris, 1898, pp. 119-120.

- BERTHO (R.P.J.) : "Perles d'aigry, d'accory ou de Popo". In Notes Africaines, n² 24, octobre 1944, pp. 1-2.

- MAUNY (R.) : "Que faut-il appeler "pierres" d'aigry?" In Notes Africaines, n 42, avril 1949, pp. 33-35.

- FAGE (J.D.) : "Some Remarks on Beads and Trade in Lower Guinea in the Sixteenth and Seventeenth centuries". In Journal of African History, III, 2, 1962, pp. 343-347.

- LAMB (H.A.) et YORK (R.N.) : "A note on Trade-Beads as Type-Fossils in Ganaian Archaeology". In West African Journal of Archaeology, 2, 1972, pp. 109-113.

- KOUAOVI (A.B.M.) : Proverbes et Dictons du Bénin. Lyon, 1981, 15 p. ill.

- QUENUM (M.) : Au pays des Fons. Us et Coutumes du Dahomey, Paris, Maisonneuve et Larose, 3 ème édit. 1983, p. 71.

- RIVALLAIN (J.) et IROKO (A.F.) : Les collections monétaires. Paris, Administration des Monnaies et Médailles, 1986, 89 p. XXXI pl.

- LAW (R.) : The Slave Coast of West Africa (1550-1750). The Impact of the Atlantic Slave Trade on an African Society.Clarendon Press, Oxford, 1991, $376 \mathrm{p}$.

2. PAULME, D.: "Perles". In MAQUET , J. : édit. Dictionnaires des civilisations africaines. Paris, Fernand Nathan, Editeur, 1968. Voir en particulier pp. 324-325.

3. Il y avait une extrême variété de perles en usage en Afrique durant la période précoloniale. Celles de verre par exemple, dont les origines sont connues n'ont pas une place dans l'univers religieux des Africains.

4. HESS, J. : L'âme nègre. Paris, 1898, pp. 119-120.

5. ELLIS, A.B., : op.cit., 1890, p. 49.

6. Idem Ibidem, 1890, p. 49.

7. DUNGLAS, E. : "Perles anciennes trouvées au Dahomey", in Première Conférence internationale des Africanistes de l'Ouest, (comptes-rendus), Dakar, IFAN, 1951, T. II, p. 432.

8. Idem, Ibidem., 1951, p. 432.

9. BURTON (Sir R.) : op.cit., 1864 (mais en deux volumes, voir la page 98 du volume II).

10. QUENUM, M. : Au pays des Fons. US et Coutumes du Dahomey, Paris, Maisonneuve et Larose, 3 ème édition, 1983, p. 71.

11. Idem, Ibidem, 1893, p. 71.

12. BERTHO, J. : "Pierres d'aigry, d'accory ou de Popo", in Notes Africaines, octobre 1944, p. 2.

13. Idem, Ibidem, 1944, p. 2.

14. BERTHO, J. op.cit., 1944, p. 2. 
15. Les perles ne sont pas portées de la même manière par les Sakpatasi que par les Hêviososi chez les Fon, par exemple.

16. C'est l'ancêtre de cette collectivité qui, seul se nommait au XVII ${ }^{e}$ siècle Gangnihèsu. Par la suite tous les chefs de sa collectivité qui sont en quelque sorte ses successeurs portent son nom dès qu'ils accèdent au trône.

17. C'est la grande divinité des Alladahonu de Huawé Zunzonsa. Les princes appartiennent à cette collectivité.

18. BERTHO (R.P.J.), op.cit., p. 2.

19. Il n'est pas rare de placer des perles dans des offrandes déposées à un carrefour à l'intention d'un esprit, d'un génie.

20. CHAMPAULT, D. et LANGLE M. de: "Notes sur l'emploi de quelques matériaux d'origine marine en Afrique du Nord" in L'Ethnographie, Nlle série, 1964-65, pp. 88-118. Voir en particulier la page 4 .

21. DUNGLAS E. : "Perles anciennes trouvées au Dahomey, 1951, p. 432.

22. DAHOMEY : ancien nom de la Colonie française aujourd'hui appelée République du Bénin.

23. Il arrive souvent qu'une partie des perles de la défunte soit partagée entre ses filles, le reste étant placé dans sa tombe. Notons au passage que les perles sont toujours beaucoup plus utilisées par les femmes que par les hommes, notamment comme parure. Il est rare, en dehors des domaines politique, religieux magique et thérapeutique, qu'un homme porte volontiers des perles comme parure.

24. N'oublions pas que nous sommes ici dans un contexte socio-culturel de croyance en la survivance de l'âme dans l'au-delà où le mort continue à mener une existence outre-tombe : "les morts ne sont pas morts" dit-on couramment en Afrique.

25. Ces statuettes - c'est bien connu - sont entretenues tout comme si elles étaient effectivement des êtres humains vivants.

26. BERTHO (R.P.J.) : "Pierres d'aigry d'accory ou de Popo", 1944, p. 2.

27. Les fon disent $f a$ et les yoruba ifa pour désigner la même réalité.

28. Voir supra, première partie de cette étude.

29. "Zone perle", "zone or", "zone cauris", etc., dans le sens où nous parlons aujourd'hui de zone franc, zone dollar, etc.

30. MOUEZY, H. : Assinie et le royaume de Kninjabo. Histoire et coutumes, Paris, Larose, 286 p. voir en particulier la page 19.

31. DELAFOSSE, M. : Essai de manuel de la langue agni parlée dans la moitié orientale de la Côte-d'Ivoire, Paris, Librairie Africaine et Coloniale, J. André, Editeur, 1901, 226 p., lire en particulier la page 37.

32. SMITH, G. : PEREIRA, D.P. : Esmeraldo de situ arbis, Lisbonne, Edit. Lisboa Imp. Nat. 1892, p. 69 et 73 .

33. BRAUN, S. : Cité par MAUNY, R. : Que faut-il appeler "pierre"s d'aigry? in Notes Africianes, $\mathrm{n}$ -42, avril 1949, p. 34.

34. DUNGLAS, E : "Perles anciennes...", 1951, p. 431.

35. Cette croyance est très répandue dans le Golfe du Bénin où de multiples précautions - dont celles à base de perles - sont prises par des marchands et surtout des marchandes pour "conjurer le regard voleur" des gens mal intentionnés.

36. Il est très bien connu que l'achat d'une marchandise avec une pièce de monnaie maléfiquement appelée et remise au vendeur provoquerait la ruine chez ce dernier qui deviendrait progressivement pauvre. Il y a encore beaucoup à faire en matière de recherche sur cette forme d'anthropologie commerciale, à notre connaissance très peu étudiée.

37. Certaines variétés de perles étaient importées d'Europe en Afrique, alors que d'autres seraient localement pêchées dans des cours d'eau. C'est ainsi que maints chercheurs, entre autres Théodore Monod, ont émis la thèse d'une telle origine à propos des fameuses perles d'aigris dont l'origine a été si controversée (R. MAUNY, op.cit., 1949, pp. 33-35). 
38. SMITH, G. : Voyage de Guinée Paris, 1751, T. II, pp. 89-91.

39. Personne dans le royaume, quelle que soit sa richesse, n'a le droit de porter le nom de Jêhosu en dehors du roi, sous peine de crime de lèse- majesté.

40. BERTHO, J. : "Coiffures-masques à franges de perles chez les rois yoruba de Nigéria et du Dahomey", in Notes Africaines, $\mathrm{n}^{\circ}$ 47, 1950, pp. 71-74.

41. L'on comprend que le R.P. Jacques BERTHO qualifie ce couvre-chef de Coiffures-masques (BERTHO), 1950, n 47, pp. 71-74.

42. Il semble qu'un tel couvre-chef fut utilisé par les rois d'Abomey à un moment donné de l'histoire de leur royaume.

43. Les yoruba appellent ce couvre-chef adé-ilèkè ou couronne de perles.

44. ADEL, S.F.: "Native crowns", in Journal of the Royal African Society, n², pp. 1902-1903, pp. 312-315.

45. Il convient de retenir que l'harmonie dans l'enfilage et l'agencement des perles est toujours recherchée dans tous ces domaines mais elle n'est jamais la priorité dominante comme dans le cas des rangées de perles utilisées comme parures.

46. Des dignitaires, à titre strictement personnel et privé, apprêtaient magiquement, à leur manière leurs colliers de perles qui sont parfois de véritables talismans protecteurs et portebonheurs mais ce n'est qu'une initiative personnelle.

47. OJO, G.J.A. : Yoruba culture. University of Ife and University of London Press Ltd, 1966, 303 p. Lire en particulier p. 259.

48. Ces pratiques, tombées en désuétude, sont aujourd'hui passées de mode, et ont disparu, notamment des milieux urbains. De très rares survivances peuvent cependant, mais de façon exceptionnelle, être observées dans des campagnes lointaines.

49. Nous sommes ici dans le domaine de la symbolique des couleurs et des interdits qui lui sont liés.

50. Ces genres de perles qui ont complètement disparu aujourd'hui de la circulation - on peut en trouver chez les femmes âgées - n'étaient jamais portées par des hommes. Elles étaient exclusivement l'affaire des femmes.

51. Les gourdes entourées de filières de perles pour la musique sont beaucoup plus utilisées dans l'aire culturelle yoruba que dans celle d'ajatado.

52. DUNGLAS, E. : "Perles anciennes...", 1951, p. 433.

53. KOUAVI, A.B.M., : Proverbes et Dictons du Bénin, Lyon, 1989, p. 100.

54. KOUAOVI, A.B.M., op.cit., 1989, p. 100.

55. Idem, Ibidem, 1989, p. 75.

56. KOUAOVI, A.B.M., op.cit., 1989, p. 75.

\section{RÉSUMÉS}

European traders selling pearls from Europe or from other parts of Africa to the blacks of Benin could not have imagined that the pearls could have been used other than ornamentally. But the Africans integrated the pearl into their different cultures, going quite beyond the superficial and immediate visual effect. Studies of these effects contribute little having lost their originality.

However, from another perspective, the study of the different non-ornamental functions of 
pearls help the researcher shed light on relevant lines of research for the historian and the ethnologist and anthropologist seeking to understand mental structures.

\section{AUTEUR}

\section{A. FÉLIX IROKO}

Université Nationale du Bénin - Département d'Histoire et d'Archéologie - Cotonou - Bénin 